\title{
Cervical Ossification of Posterior Longitudinal Ligament in X-Linked Hypophosphatemic Rickets Revealing Homogeneously Increased Vertebral Bone Density
}

\author{
Masato Shiba, Masaki Mizuno, Keita Kuraishi, Hidenori Suzuki \\ Department of Neurosurgery, Mie University Graduate School of Medicine, Tsu, Japan
}

There is no report that describes in detail the radiological and intraoperative findings of rickets with symptomatic cervical ossification of the posterior longitudinal ligament. Here, we describe a case of X-linked hypophosphatemic rickets with cervical ossification of the posterior longitudinal ligament presenting unique radiological and intraoperative findings. The patient presented progressive tetraparesis. Magnetic resonance imaging studies revealed severe cervical spinal cord compression caused by ossification of the posterior longitudinal ligament. Computed tomography scans revealed homogeneously increased vertebral bone density. An expansive laminoplasty was performed. At surgery, homogeneously hard lamina bone was burdened in drilling and opening of the laminae. The patient's neurological symptoms were improved postoperatively. Bony fusion of the hinges occurred postoperatively. Therefore, expansive laminoplasty could be performed for symptomatic cervical ossification of the posterior longitudinal ligament with X-linked hypophosphatemic rickets. However, unusual bone characters should be taken into consideration for careful operation during surgery.

Keywords: Laminoplasty; Rickets; Ossification of posterior longitudinal ligament

\section{Introduction}

$\mathrm{X}$-linked hypophosphatemic rickets (XLHR) is characterized by X-linked dominant pattern of inheritance and hypophosphatemia associated with decreased renal tubular reabsorption of phosphate [1]. Spinal canal stenosis and spinal cord compression caused by thickening of the laminae, hypertrophy of the spinal ligaments, or facet joints in XLHR [2] are rare. Here we report a case of XLHR with severe cervical spinal cord compression caused by ossification of the posterior longitudinal ligament (OPLL) with unique radiological and intraoperative findings.

\section{Case Report}

A 32-year-old man was referred to our hospital due to a 1-year progressive sensorimotor disturbance to the extremities. He had been diagnosed with vitamin Dresistant rickets and received $1 \alpha$-hydroxyvitamin $\mathrm{D}_{3}$ $\left[1 \alpha-(\mathrm{OH})-\mathrm{D}_{3}\right](1 \mu \mathrm{g} /$ day $)$ since his childhood. His mother, older sister, nephew and daughter also had rickets (Fig. 1). He was of short stature $(151.0 \mathrm{~cm})$ with limb shorten-

Received Jun 21, 2014; Revised Jul 29, 2014; Accepted Aug 7, 2014

Corresponding author: Masato Shiba

Department of Neurosurgery, Mie University Graduate School of Medicine,

2-174 Edobashi, Tsu, Mie 514-8507, Japan

Tel: +81-59-232-1111, Fax: +81-59-231-5212,E-mail: m_shiba1206@yahoo.co.jp 
ing. Neurological examination revealed numbness below T4, weakness (manual muscle testing, 4/5; grip strength: right $17.0 \mathrm{~kg}$, left $13.0 \mathrm{~kg}$ ), deep tendon hyper-reflexes of the upper and lower extremities, and spastic gait disturbance needing assistance, comparable to the Japanese Orthopaedic Association (JOA) score [3] of 7 points. His serum phosphate level $(2.0 \mathrm{mg} / \mathrm{dL}$; normal range, $2.3-4.5$ $\mathrm{mg} / \mathrm{dL}$ ) was low, whereas his alkaline phosphatase (726 units/L; normal range, 100-350 units/L) and high sensitivity parathyroid hormone levels $(770 \mathrm{pg} / \mathrm{mL}$; normal range, $160-520 \mathrm{pg} / \mathrm{mL}$ ) were high. His serum levels of

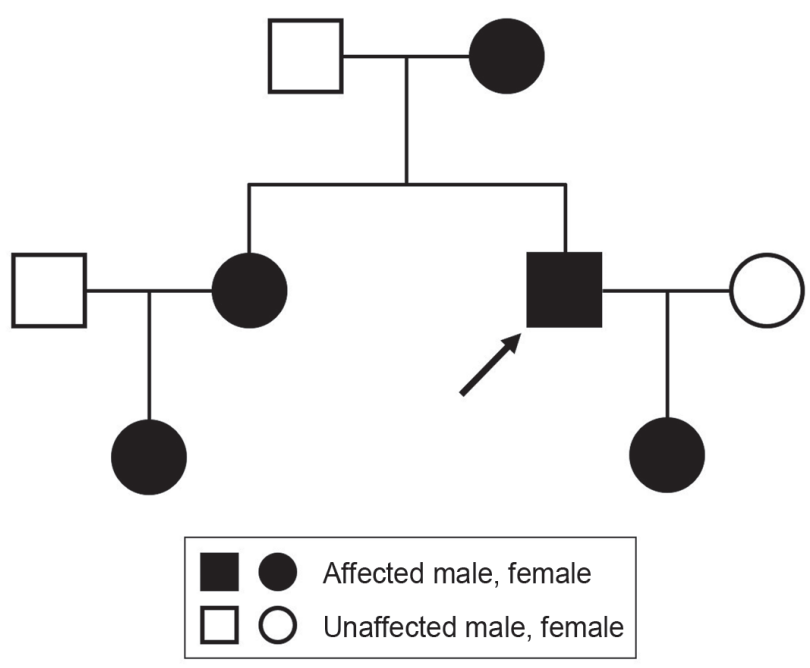

Fig. 1. Family tree of the patient in our case. The present case is indicated by an arrow. calcium, intact-parathyroid hormone, growth hormone, insulin-like growth factor, 125-hydroxyvitamin $\mathrm{D}_{3}$, and 1 , 25-dihydroxyvitamin $\mathrm{D}_{3}\left[1,25-(\mathrm{OH})_{2}-\mathrm{D}_{3}\right]$ were all within normal ranges. Plain radiography and computed tomography $(\mathrm{CT})$ of the cervical spine demonstrated a mixed type of OPLL from C2 to C7 (Fig. 2A-C). Magnetic resonance imaging (MRI) demonstrated cervical spinal canal stenosis and spinal cord compression at the $\mathrm{C} 2 / 3$ to $5 / 6$ levels. The compression was prominent the most at the C4/5 level with intramedullary high signal intensity on T2-weighted images (Fig. 2D). CT also demonstrated generalized bone density increase, mimicking osteopetrosis (Fig. 2A-C). Although a genetic examination was not performed, a diagnosis of XLHR was made based on his family history, a predominance of females affected, hypophosphatemia, and normocalcemia. Cervical myelopathy due to OPLL was treated with expansive laminoplasty.

The patient was placed in the prone position. His head was fixed with protective helmet system (Prone View, Dupaco, San Diego, CA, USA) which holds the cushion molding a head/face, because his cranial bone was too thin on CT to be fixed with a head-pin frame (Fig. 2E). C2 dome-like laminotomy as well as C3-6 mid-sagittal splitting and lateral guttering were performed using highspeed 2-4 mm-diameter diamond burr under the surgical microscope. His lamina bones were homogeneously hard. Characteristic of cancellous bone was not observed. Thus, the inner cortex was thinned carefully according to
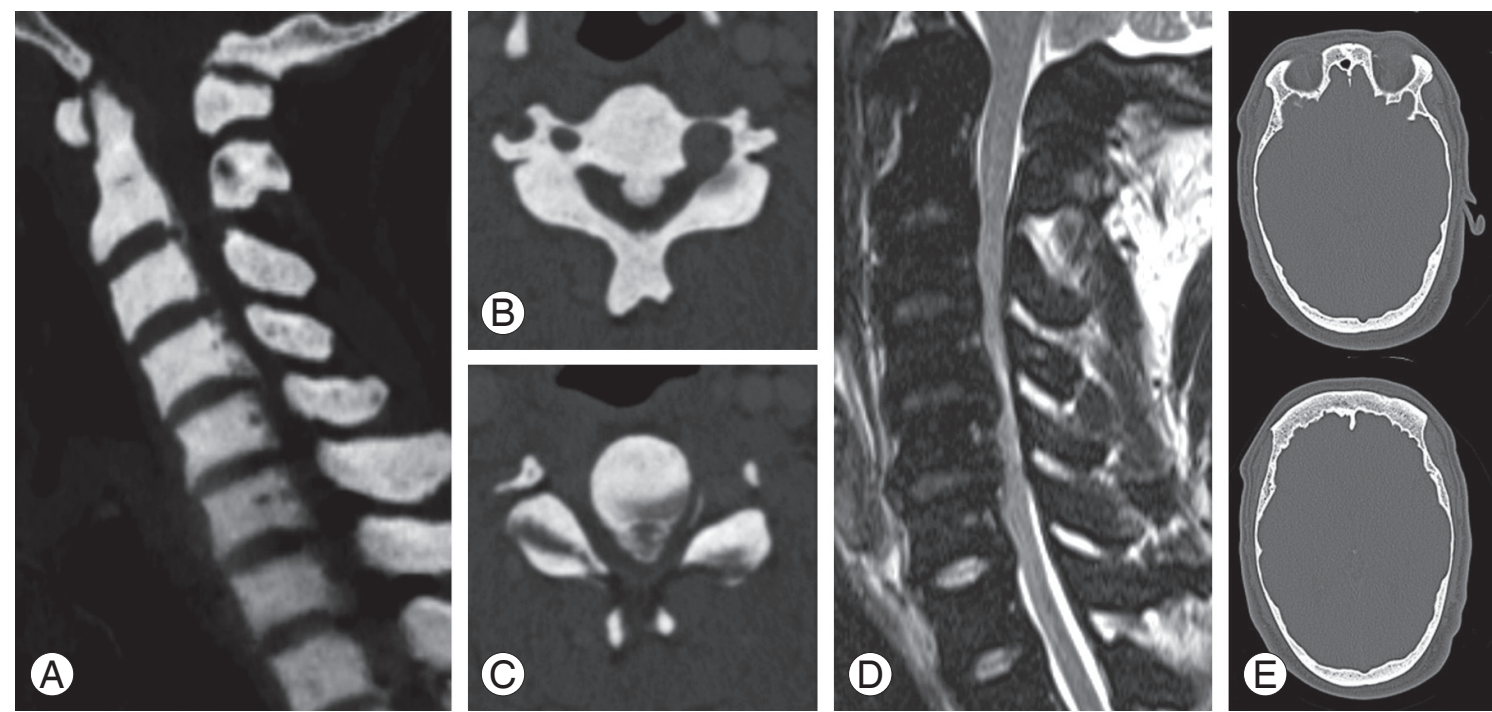

Fig. 2. Preoperative computed tomography (CT) scan demonstrating a cervical ossification of the posterior longitudinal ligament (A). Mass of the ossification of the posterior longitudinal ligament is more prominent at the C4 (B) to C4/5 levels (C). T2-weighted magnetic resonance imaging demonstrating spinal canal stenosis and spinal cord compression (D). Axial section of head CT scan demonstrating a thinned cranial bone (E). 
the evaluation and measurement by preoperative images. The split laminae were opened at the hinge with unusual strong elasticity in a sequence like a French door using a laminar spreader. After dural pulsation of the cord was observed, hydroxyapatite spacers (APACERAM, PENTAX, Tokyo, Japan) were inserted between the laminae from C3 through C6 using flexible titanium cables (Sof' wire system, Codman, Raynham, MA, USA) (Fig. 3).

He was treated with oral phosphate supplementation
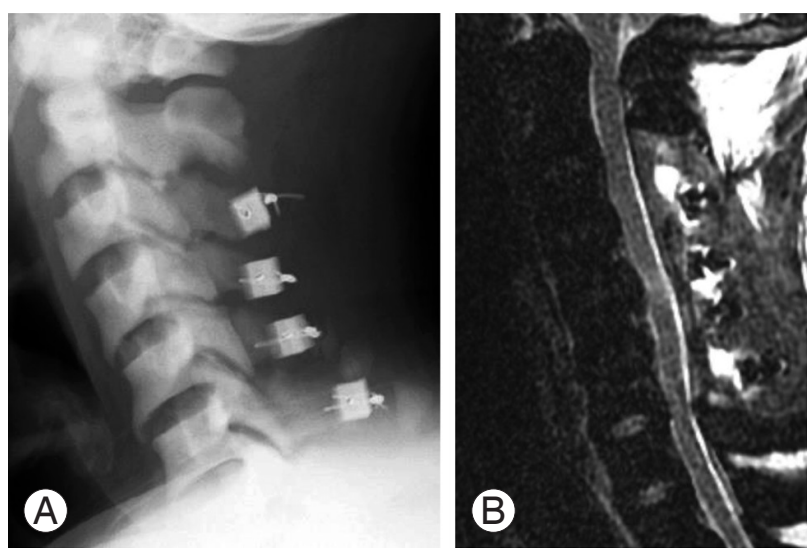

Fig. 3. Postoperative plain radiograph (A) and T2-weighted magnetic resonance imaging $(\mathbf{B})$ demonstrating adequate expansion of the spinal canal and decompression to the spinal cord.
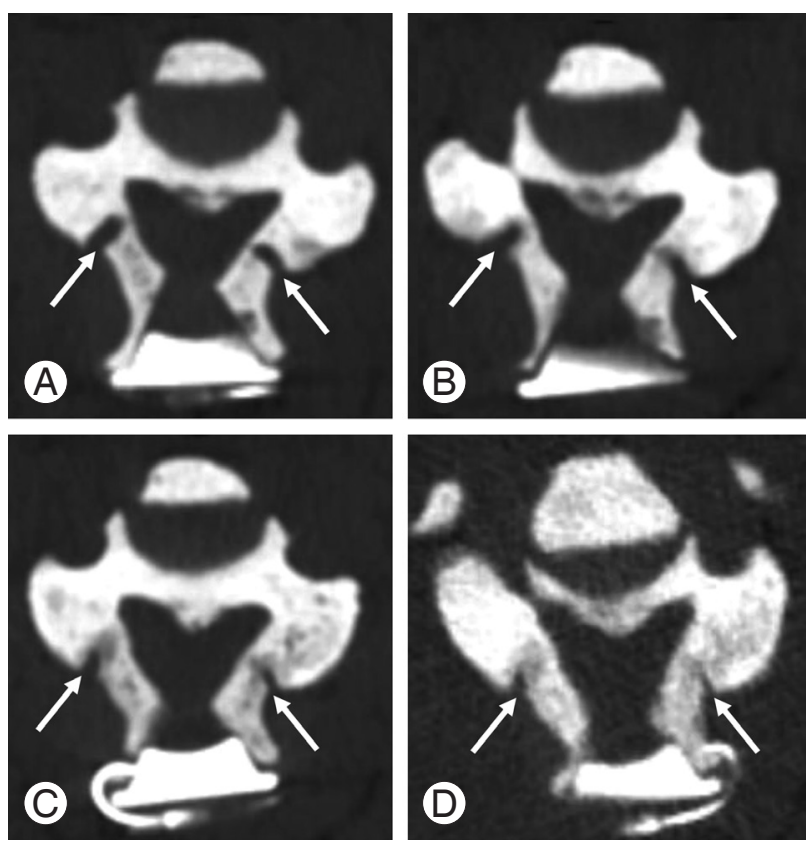

Fig. 4. Postoperative computed tomography scans at C4 obtained after the surgery demonstrating bony fusion of the hinges of the expanded laminae (arrows) with gradual advances. (A) Immediately after surgery. (B) 4 months after surgery. (C) 7 months after surgery. (D) 14 months after surgery. since the time of the operation in addition to taking $1 \alpha-(\mathrm{OH})-\mathrm{D}_{3}(1 \mu \mathrm{g} / \mathrm{day})$. Six months after the operation, his sensorimotor disturbance disappeared. His grip strengths were $29.0 \mathrm{~kg}$ bilaterally. He could go up and down stairs independently with a clutch (JOA score, 14 points; the recovery rate, $57.1 \%)$. CT scans revealed progressive bony fusion of the hinges of expanded laminae at 4, 7, and 14 months after the operation (Fig. 4).

\section{Discussion}

XLHR is caused by a loss-of-function mutation in the PHEX gene (phosphate regulating gene with homologies to endopeptidases on the $\mathrm{X}$ chromosome) located on $\mathrm{X}$ chromosome Xp22.1. XLHR is characterized by inadequate mineralization of bone [4] due to high incidence of hyperparathyroidism [5]. Marked shortening of legs in XLHR might be due to the reduction of mineral content in the peripheral bone [5]. In contrast to peripheral bones, cancellous bone density and mineralization are increased in axial bone, including lumbar spine [5]. However, there is no report of a case exhibiting extremely predominant increase in cancellous bone density of the cervical spine on plain X-rays and CT scans in adult XLHR with cervical OPLL as seen in the present case. The cranial bone thinning observed in this case might have been caused by inadequate bone mineralization in XLHR.

Intraoperative findings in previous reports revealed thickened and hard lamina [6,7], ossified ligamentum flavum and posterior longitudinal ligament [8], and heavily calcified anulus fibrosus [2]. The lamina bones were also hard in this present case. However, intraoperative findings revealed that homogeneously mineralized bone was continued through the lamina and that the characteristic of cancellous bone was absent. In developmental spinal canal stenosis, the cortical bone exists as an obvious different structure in the spinal canal side after the cancellous bone of the lamina is drilled out. In rickets as shown in the present case, the boundary between cortical and cancellous bones might be unclear. Thus, drilling with diamond burr and opening of the lamina were performed in a meticulous fashion. Although there was no mention of using a microscope in previous reports [2,6-8], we consider that it is essential to use a microscope during drilling of the lamina to prevent injury to the facet joints and the spinal cord. As to the method of laminoplasty, it is currently unknown whether mid-splitting or open 
door method is better for our case. We selected mid-splitting laminoplasty because we were more familiar with it. There was no report on open door laminoplasty for cervical OPLL in XLHR.

The bone bonding rate of hinges of the expanded laminae with hydroxyapatite ceramic spacers has been reported to be $84 \%$ at 6 months and $98 \%$ at 1 year postoperatively in cased of developmental spinal canal stenosis [9]. However, the rate in XLHR has not been reported. Both activated vitamin $\mathrm{D}$ and phosphate are recommended for affected adults with spontaneous insufficiency fractures and pending orthopaedic procedures to reduce recovery time [10]. This study showed that bony fusion of the hinges occurred even in XLHR patient who received proper oral phosphate supplementation and vitamin $\mathrm{D}$ treatment.

In conclusion, although spinal cord compression with cervical OPLL is a rare complication in XLHR, surgery is a treatment of choice for symptomatic patients. During surgery, unusual bone characters should be taken into consideration for careful operation.

\section{Conflict of Interest}

No potential conflict of interest relevant to this article was reported.

\section{References}

1. Durmaz E, Zou M, Al-Rijjal RA, et al. Novel and de novo PHEX mutations in patients with hypophosphatemic rickets. Bone 2013;52:286-91.

2. Soehle M, Casey AT. Cervical spinal cord compression attributable to a calcified intervertebral disc in a patient with X-linked hypophosphatemic rickets: case report and review of the literature. Neurosurgery 2002;51:239-42.

3. Hirabayashi K, Miyakawa J, Satomi K, Maruyama T, Wakano K. Operative results and postoperative progression of ossification among patients with ossification of cervical posterior longitudinal ligament. Spine (Phila Pa 1976) 1981;6:354-64.

4. Oliveri MB, Cassinelli H, Bergada C, Mautalen CA. Bone mineral density of the spine and radius shaft in children with X-linked hypophosphatemic rickets (XLH). Bone Miner 1991;12:91-100.

5. Reid IR, Murphy WA, Hardy DC, Teitelbaum SL, Bergfeld MA, Whyte MP. X-linked hypophosphatemia: skeletal mass in adults assessed by histomorphometry, computed tomography, and absorptiometry. Am J Med 1991;90:63-9.

6. Bradbury PG, Brenton DP, Stern GM. Neurological involvement in X-linked hypophosphataemic rickets. J Neurol Neurosurg Psychiatry 1987;50:810-2.

7. Yoshikawa S, Shiba M, Suzuki A. Spinal-cord compression in untreated adult cases of vitamin-D resistant rickets. J Bone Joint Surg Am 1968;50:743-52.

8. Lee SH, Agashe MV, Suh SW, et al. Paravertebral ligament ossification in vitamin D-resistant rickets: incidence, clinical significance, and genetic evaluation. Spine (Phila Pa 1976) 2012;37:E792-6.

9. Tanaka N, Nakanishi K, Fujimoto Y, et al. Expansive laminoplasty for cervical myelopathy with interconnected porous calcium hydroxyapatite ceramic spacers: comparison with autogenous bone spacers. J Spinal Disord Tech 2008;21:547-52.

10. Carpenter TO, Imel EA, Holm IA, Jan de Beur SM, Insogna KL. A clinician's guide to X-linked hypophosphatemia. J Bone Miner Res 2011;26:1381-8. 